\title{
Levantamento etnobotânico de plantas medicinais utilizadas pela comunidade de Nacuale, no Parque Nacional das Quirimbas, Moçambique
}

\author{
Alexandre Jacinto MUCHAIA ${ }^{*}$, Salvador José António NANVONAMUQUITXO² \\ ${ }^{1}$ Bioserve Limitada, Pemba, Cabo Delgado, Moçambique. \\ ${ }^{2}$ Faculdade de Ciências Naturais da Universidade Lúrio, Pemba, Cabo Delgado, Moçambique. \\ *E-mail: alexandremuchaia@gmail.com \\ (ORCID: 0000-0001-5536-8644; 0000-0001-9354-5760)
}

Recebido em 28/06/2021; Aceito em 17/12/2021; Publicado em 28/12/2021.

\begin{abstract}
RESUMO: A presente pesquisa teve como objectivo fazer o levantamento Etnobotânico de plantas com poder medicinal utilizadas pela comunidade de Nacuale no Distrito de Ancuabe, Cabo Delgado, Moçambique. Foram realizadas entrevistas com 88 chefes de agregados familiares com propósito de compreender além do perfil socio -económico, as principais plantas usadas para o tratamento de doenças mais frequentes da comunidade desta localidade. As plantas citadas como medicinal foram identificadas e organizadas em função dos valores de concordância de uso principal-CUPc. Por sua vez, foi determinada a diversidade de plantas medicinais usadas na região, bem como as doenças para a qual cada uma das plantas é usada. Cerca de $45 \%$ da população que vive na localidade de Nacuale é pobre (rendimento familiar inferior a $15 \$ /$ mês). As principais actividades de renda são a agricultura familiar $(68 \%)$, caça $(20 \%)$ e pequenos negócios $(12 \%)$. O difícil acesso aos serviços de saúde convencional faz com que a maioria das populações optem pela medicina tradicional para tratamento de doenças. A Mangifera indica Wall (46,0\%), Afizelia quanzensis Welw (37,5\%), Moringa oleifera Lam (29,2\%) e Carica papaya L $(20,8 \%)$ foram as espécies mais citadas dentre as utilizadas para o tratamento de doenças. As populações demonstraram ainda ter conhecimento de diversas espécies de plantas medicinais da região $\left(\mathrm{H}^{\prime}=3,04\right.$ e J'=0,93), enquanto que as doenças comumente tratadas com recurso destas plantas são a malária $(20 \%)$, dores de estomago (18\%), dores de cabeça (16\%) e reumatismo (13\%). Na localidade de Nacuale, o uso da medicina tradicional é ainda uma opção viável pela dificuldade de acesso aos serviços de saúde convencional, agravado pelo baixo nível de escolaridade, pobreza e aspectos socioculturais. As plantas são usadas para tratamento de doenças mais frequentes nessa região.
\end{abstract}

Palavra chaves: etnobotânica; plantas medicinais; Cabo Delgado - Moçambique.

\section{Ethnobotanical assessment of medicinal plants used by the community of Nacuale, in the Quirimbas National Park, Mozambique}

\begin{abstract}
The objective of the present research was to conduct an ethnobotanical survey of plants with medicinal uses in the village of Nacuale in Ancuabe District, Cabo Delgado, Mozambique. 88 households were interviewed with the purpose of understanding, in addition to the socio-economic profile, the most common plants used for the treatment of the community's most frequent diseases in this village. The plants cited as medicinal were identified and organized according to the values of concordance of principal use-CUPc. In addition, the diversity of medicinal plants used in the region was determined, as well as the diseases for which each of the plants is used. About $45 \%$ of the population living in Nacuale is low-income (household income less than $15 \$ /$ month). The main income generating activities are subsistence farming (68\%), hunting (20\%) and small businesses $(12 \%)$. The difficult access to conventional health services makes most of the population opt for traditional medicine for the treatment of diseases. Mangifera indica Wall (46.0\%), Afizelia quanzensis Welw (37.5\%), Moringa oleifera Lam $(29.2 \%)$, Carica papaya L $(20.8 \%)$ were cited as the species most used for the treatment of these diseases. The populations also demonstrated knowledge of several species of medicinal plants of the region $\left(\mathrm{H}^{\prime}=3.04\right.$ and $\left.\mathrm{J}^{\prime}=0.93\right)$, and the diseases commonly treated with these plants are malaria $(20 \%)$, stomach aches $(18 \%)$, headaches $(16 \%)$ and rheumatism $(13 \%)$. Thus, in the village of Nacuale the use of traditional medicine is still a viable option due to the difficulty of access to conventional health services, recorded by the low level of education, poverty, and socio-cultural aspects. The plants are used to treat the most frequent diseases in the region.
\end{abstract}

Keywords: ethnobotany; medicinal plants; Cabo Delgado - Mozambique.

\section{INTRODUÇÃO}

As plantas medicinais são todos os vegetais que contêm na sua estrutura ou em seus órgãos, substâncias que podem ser terapêuticas e/ou utilizadas como matéria-prima na síntese de compostos químicos farmacêuticos (WORLD HEALTH ORGANIZATION-WHO, 2002).
O uso de plantas medicinais para além de ser uma prática milenar, tem apoiado as comunidades no enfrentamento de diversos obstáculos do processo evolutivo até aos dias actuais (AGOSTINHO; DA SILVA, 2012). Esta relação entre as plantas e o homem, comumente denominada por etnobotânica (FONSECA-KRUEL; PEIXOTO, 2004; 
GIRALDI, 2010), tem sido orientada por uma série de conhecimentos acumulados ao longo do tempo e que são transmitidos oralmente entre as gerações (CATELLUCCI et al., 2000).

Actualmente a etnobotânica tornou-se mais expressiva na medicina, principalmente em regiões menos desenvolvidas, como na África, em que cerca de $80 \%$ da população utiliza a medicina tradicional para suprir as suas necessidades de saúde (CUNNINGHAM, 1993; HOSTETTMANN et al., 2000; WHO, 2002).

Em Moçambique, as pesquisas relacionadas com plantas medicinais começaram a apresentar maior expressão nos anos 70, com a criação do Gabinete de Investigação da Medicina Tradicional no Ministério da Saúde (MISAU), e nas décadas de 80 à 90 com a criação da Associação de Médicos Tradicionais de Moçambique - AMETRAMO (AGOSTINHO; DA SILVA, 2012). Em 2008, foi criado o Centro de Investigação e Desenvolvimento em Etnobotânica, com a finalidade de expandir os cuidados de saúde e garantir o acesso seguro, racional e sustentável das plantas medicinais (CONDE et al., 2014).

Embora a criação destes núcleos tenha despertado interesses em pesquisas nesta temática (OLSEN, 2006; ERNESTO, 2006; SERRANO et al., 2008; PINTÃO; SILVA, 2008; GOMES; SILVA, 2009; SIMBINE, 2013), os estudos até então realizados, se concentram mais nas regiões Sul e Centro do país, criando ainda uma enorme lacuna de informações sobre plantas medicinais utilizadas pelas comunidades da região Norte, como é o caso da localidade de Nacuale no Distrito de Ancuabe, na Província de Cabo Delgado.

Nacuale, apresenta uma rede sanitária pouco abrangente para a comunidade rural (MAE, 2014), sendo o uso de plantas medicinais extremamente importante para o tratamento de doenças. No entanto, nesta região, como na maioria da região Norte do país, ainda não existem registos documentais sobre as plantas usadas para tratamento de doenças.

Segundo Castro; Ferreira (2001), as pesquisas sobre plantas medicinais permitem que a informação sobre esta temática, que é passada de geração para geração de forma oral, seja documentada, reduzindo assim a possibilidade de extinção de conhecimento sobre o uso de inúmeras espécies, muitas das quais ainda desconhecidas pela ciência. Por esta razão, Agostinho (2016) afirma que estes estudos devem ser considerados urgentes e de extrema importância, principalmente em regiões onde ainda é usada a medicina tradicional.

Esse artigo objectivou apresentar o levantamento etnobotânico de plantas com poder medicinal utilizadas pela comunidade de Nacuale (Parque Nacional das Quirimbas), no Distrito de Ancuabe, Província de Cabo Delgado, Norte de Moçambique.

\section{MATERIAL E MÉTODOS}

\subsection{Caracterização da área de estudo}

O estudo foi realizado na localidade de Nacuale (nas comunidades de Ungura, Miegane e Muela) no Distrito de Ancuabe, região Sul da zona tampão do bloco A, do Parque Nacional das Quirimbas (MINISTERIO DE ADMINISTRAÇÃO ESTATAL-MAE，2014). O Parque está localizado na região norte de Moçambique com uma área estimada em cerca de 750639 ha, dos quais $80 \%$ representam habitats de terra firme e os restantes $20 \%$ habitats marinhos e ilhéus (PARQUE NACIONAL DAS QUIRIMBAS-PNQ, 2016).

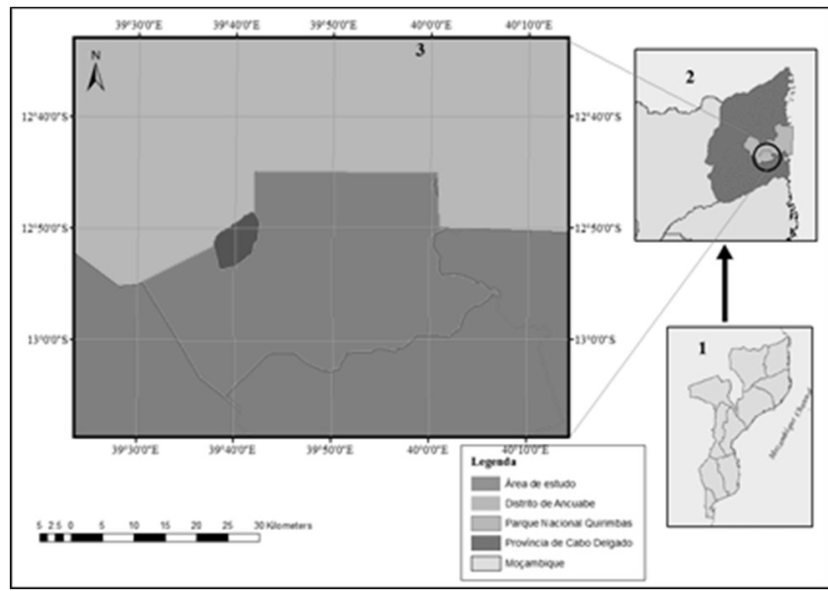

Figure 1. Localização da área de estudo na zona tampão do PNQ no distrito de Ancuabe província de Cabo Delgado- Moçambique Figure 1. Location of the study area in the buffer zone of the PNQ in the district of Ancuabe province of Cabo Delgado- Mozambique

A região apresenta clima semi-árido e sub-húmido seco, com duas épocas (chuvosa e seca) bem definidas; a precipitação média anual varia de 800 a $1200 \mathrm{~mm} \mathrm{e}$ as temperaturas médias anuais variam entre $20^{\circ}$ a $25^{\circ} \mathrm{C}$. A região possui vários rios, quase na sua maioria de regime intermitentes, sendo considerados como principais o rio Muaguide, o rio Montepuez e o rio Megaruma (MAE, 2014).

O tipo florestal característico desta região é comumente denominado por Miombo. Miombo é um termo coloquial típico da região subsaariana da África, usado para caracterizar a tipologia florestal dominada por espécies da família Fabaceae, com maior enfoque nas espécies do género Brachystegia, Jubernardia e Isoberlina (NANVONAMUQUITXO et al., 2019).

De acordo com Bandeira et al. (2007), as principais espécies dominantes nesta região são: Cenostigma tocantinum Ducke, Milletia stublmanni Taub, Afzelia quanzensis Welw, Pterocarpus angolensis DC, Aniba rosaeodora Ducke, Caesalpinia leiostachya (Benth.) Ducke, Khaya nyasica Stapf ex Bakerf., Albizia gummifera C.A.Sm., Adansonia digitata L, Sterculia appendiculata K.Schum., Bombax rhodognaphalon, Bombax rhodognaphalon K.Schum., Cordyla africana Lour., Annona senegalensis pers e Combretum zeyheri Sond. A fauna da região é bastante diversificada, podendo se encontrar com frequência grupo de carnívoros, répteis, anfíbios, herbívoros e aves (MAE, 2014).

A agricultura é a principal actividade económica para a maior parte das comunidades desta região, sendo caracterizada por uma produção de pequena escala, somente para atender as necessidades básicas do agregado familiar. As principais culturas agrícolas resultantes desta actividade são mandioca, milho, mapira, feijões, amendoim e ameixoeira (INSTITUTO NACIONAL DE ESTATÍSTICA-INE, 2013).

\subsection{Coleta de dados}

A colecta de dados seguiu o processo de amostragem estratificada, sendo que cada comunidade foi considerada como sendo um estrato, conforme Monteiro (2012). Foi considerado o tamanho da população de 1105 agregados familiares, sendo determinado o tamanho da amostra pela equação 1, conforme recomendações de Richardson (1999). 


$$
\mathrm{n}=\frac{Z^{2} * P * q * N}{\varepsilon^{2} *(N-1)+Z^{2} * P * q}
$$

em que: n-Tamanho mínimo da amostra; N- Tamanho da população; z - Valor crítico obtido a partir do nível de confiança de 95\% (1,96); $\varepsilon$ - Erro de estimação (5\%); p- Proporção da amostra com a característica pesquisada (50\%); q- Proporção da amostra sem a característica pesquisada $(50 \%)$.

Dentro de cada estrato, aplicou-se a técnica de bola de neve para selecção dos agregados, como recomendado por Baldin; Munhoz (2011) e Vinuto (2014). Dos agregados familiares seleccionados, apenas os seus chefes foram submetidos a uma entrevista através de um questionário constituídos por duas seções de questões. A primeira seção foi composta por questões que definiam o perfil socioeconómico dos agregados familiares entrevistados e a segunda seção foi composta por questões que visavam obter informações sobre as plantas medicinais usadas para tratamento das doenças no agregado familiar.

\subsection{Análise de dados}

A caracterização do perfil sócio-económico dos agregados familiares da área de estudo compreendeu a apresentação de informações referentes ao tamanho médio de agregados, principais fontes de renda e nível de escolaridade, bem como sua relação com o uso de plantas medicinais.

A selecção das plantas medicinais mais utilizadas pelas comunidades da área de estudos foi baseada na aplicação da técnica de concordância de uso principal corrigido (CUPc), que relaciona a concordância de uso principal (CUP) e o factor de correcção (FC) (VENDRUSCOLO; MENTZ, 2006) conforme equação 2.

$$
\text { CUPc }=\text { CUP } \times \text { FC }
$$

em que: $\quad$ CUP $=\frac{\mathrm{n}^{\circ} \text { de informantes que indicaram usos principais }}{\mathrm{n}^{\circ} \text { de informantes que indicaram uso da espécies }} \times 100$ $\mathrm{FC}=\frac{\mathrm{n}^{\circ} \text { de informantes que citaram a espécie }}{\mathrm{n}^{\circ} \text { de informantes que citaram a especie mais citada }}$

As principais plantas medicinais utilizadas pela comunidade de acordo com o valor de CUPc foram apresentadas em uma tabela categórica conforme a ordem decrescente dos valores de CUPc.

As plantas citadas pelos agregados familiares como sendo as mais usadas para fins medicinais foram identificadas até ao nível de espécie, através de guias de identificação botânica comumente na região (DHARANI, 2002; BANDEIRA et al., 2007; DHARANI; YENESEW, 2010; VAN WYK; VAN WYK , 2013). A grafia e a validação dos nomes científicos das espécies identificadas foram conferidas e actualizadas taxonomicamente com base nas informações do International Plant Names Index (versão on-line 2020) e as abreviaturas estandardizadas dos nomes de autores de acordo com (BRUMMITTY; POWELL, 1992). A nomenclatura das espécies seguiu a proposta do grupo Angiosperm Philogeneny Group (APG IV, 2016).

A diversidade de plantas medicinais encontradas na área de estudo foi determinada através do Índice de ShannonWiener e do índice de Pielou (PINTO et al., 2006; SANTOS et al., 2018), conforme equações 5 e 6.

$$
\begin{aligned}
& \mathrm{H}^{\prime}=-\sum_{\mathrm{n}=1}^{\mathrm{s}}(\mathrm{Pi})(\ln \mathrm{Pi}) \\
& \mathrm{J}^{\prime}=\mathrm{H}^{\prime} / \ln S
\end{aligned}
$$

em que: $H^{\prime}=$ índice de Shannon-Wiener; Pi= Proporção de espécie dada por ni/N; ni= número de citações por espécie (considerou-se apenas uma citação por espécie por informante, mesmo que um informante tenha citado uma espécie várias vezes, para vários usos); $\mathrm{N}=$ número total de citações; J'= Índice de Pielou e $\mathrm{S}=$ número de espécies encontradas na área de estudo

As principais doenças tratadas com recurso das plantas medicinais citadas foram registadas e apresentadas em um gráfico de barras.

\section{RESULTADOS}

Foram realizadas 88 entrevistas, sendo destas 58 e $42 \%$ com chefes do AF do sexo masculino e feminino, respectivamente. As entrevistas foram realizadas nas comunidades de Ungura (56\%), Miegane (33\%) e Muela $(11 \%)$. A idade média dos chefes e o tamanho dos agregados familiares entrevistados foram de 46 anos e 5 pessoas, respectivamente. $\mathrm{O}$ nível de educação nas três comunidades é relativamente baixo, sendo que mais da metade (55\%) dos entrevistados não terminaram o nível primário. A agricultura $(68 \%)$, caça $(20 \%)$ e pequenos negócios $(12 \%)$ são as principais fontes de renda das três comunidades. Também se observou que apenas $10 \%$ das famílias conseguem obter renda superior a $185 \$ /$ mês e $45 \%$ das famílias obtêm uma renda inferior a $15 \$ /$ mês.

Nas três comunidades estudadas observou-se ausência de unidades sanitárias, assim as populações tem de percorrer cerca de 3 horas de tempo, de bicicletas para ter acesso aos serviços de saúde convencional no centro de saúde mais próximo. Este fato reflecte na situação de que 36\% das famílias dessas comunidades recorram com frequência aos médicos tradicionais, que normalmente distam cerca de 15 a 20 minutos de caminhadas.

Cerca de 50 espécies, de 21 géneros e 23 famílias botânicas foram citadas pelos agregados familiares como sendo utilizadas como plantas medicinais nas três comunidades. A Mangifera indica Wall (46,0\%), Afizelia quanzensis Welw (37,5\%), Moringa oleifera Lam (29,2\%) e Carica papaya L. (20,8\%) foram as espécies citadas utilizadas como as principais plantas medicinais (Tabela 1). Apesar disso, a família Fabaceae apresentou maior número de espécies entre as mais citadas pelas comunidades como sendo medicinal (Tabela 2).

Os índices de diversidade Shannon-Wiener ( $\left.\mathrm{H}^{\prime}\right)$ e equabilidade de Pielou (J') foram de 3,04 e 0,93, respectivamente, demostrando alta diversidade de espécies com poder medicinal usadas na região. Estas plantas são empregadas para tratar doenças e/ou sintomas mais frequentes nos agregados familiares como são os casos da malária $(20 \%)$, dores de estômago $(18 \%)$, dores de cabeça $(16 \%)$ e reumatismo $(13 \%)$ (Figura 2$)$.

\section{DISCUSSÃO}

O baixo nível de escolaridade observado nesta região está relacionado com a dificuldade que o governo local tem de expandir a educação convencional para as regiões rurais. Desta forma os membros da comunidade têm de percorrer longas distâncias para ter acesso à educação do nível secundário e/ou pré-universitário. Por outro lado, a maioria 
dos agregados familiares desta região professam a religião islâmica, e, dessa forma, estes priorizam a educação religiosa em relação a educação convencional.

A maioria dos agregados familiares desenvolvem apenas uma actividade de renda, que geralmente é a agricultura familiar, sendo que somente em períodos de seca prolongada, em que a actividade agrícola é pouco produtiva, recorrem a caça e a pequenos negócios informais, que também são pouco rentáveis, por esta razão, os rendimentos económicos da maioria dos agregados familiares são relativamente baixos.

Nesta localidade a medicina tradicional ainda é uma opção viável pelo facto das populações terem de percorrer longas distâncias para ter acesso aos serviços de saúde convencional. Por outro lado, o baixo nível de escolaridade, a pobreza e aspectos socioculturais também contribuem para o uso frequente da medicina tradicional.

As quatro espécies mais usadas para o tratamento de doenças nestas comunidades estão directamente relacionadas com as doenças mais frequentes na região (malária, dor de barriga, dores de cabeça e reumatismo), e que são geralmente tratadas com recurso a essas plantas nas zonas rurais (CLIFF et al. 2003; MAZIVE et al., 2009). A abundância de espécies da família Fabaceae na lista das espécies citadas pelas comunidades, está relacionada com a característica da vegetação da região (Miombo), dominada pelas espécies desta a família (NANVONAMUQUITXO et al., 2019), corroborando com SILVA et al. (2011)
Os valores dos índices de diversidades demonstram que as comunidades desta região dispõem de conhecimento sobre uma considerável diversidade de plantas medicinais actualmente em uso, sendo essa condição também observada em outras pesquisas similares no país (DE MELLO AMOROZO, 2002).

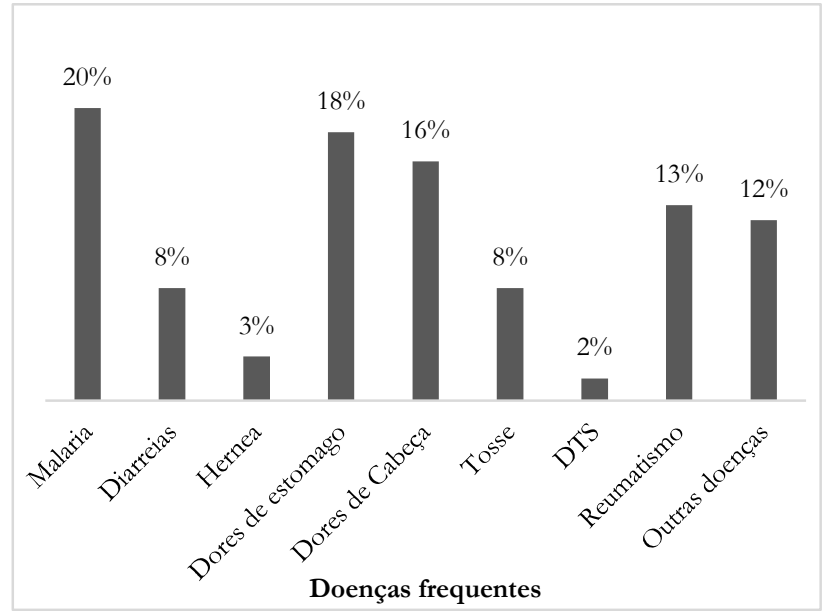

Figura 2. Doenças mais frequentes na localidade de Nacuale no PNQ, Moçambique.

Figure 2. Most frequent diseases in the locality of Nacuale in the PNQ, Mozambique.

Tabela 1. Principais plantas medicinais utilizadas para tratamento de doenças da localidade de Nacuale no PNQ, Moçambique. Table 2. Main medicinal plants used to treat diseases in the Nacuale locality in the PNQ, Mozambique.

\begin{tabular}{|c|c|c|c|}
\hline Nome Científico & Doença / sintoma Curado & Órgão da planta & CUPc \\
\hline Mangifera indica Wall. & Dores de estômago; Malária & Raíz e Folhas & 46.0 \\
\hline Afizelia quanzonsis Welw & Dores de estômago & Raíz, Caule e Folhas & 37.5 \\
\hline Moringa oleifera Lam & Dores de estômago; Malária & Raíz, Caule e Folhas & 29.2 \\
\hline Carica papaya L. & Dores de estômago; Diarreias & Raíz e Folhas & 20.8 \\
\hline Flacourtia indica (Burm.f.) Merr & Dores de estômago e Dores de vista & Raíz, Caule e Folhas & 20.8 \\
\hline Anacardium occidentale L. & Disenteria & Raiz, Caule e Folhas & 16.7 \\
\hline Psidium guajava L (GCI) & Dores de estômago; Disenteria & Raíz, Caule e Folhas & 16.7 \\
\hline Erytbrina abyssinica Lam. ex DC. & Dores de estômago; Dores de dentes; Dores de vista & Raíz, Caule e Folhas & 16.7 \\
\hline Acácia senegal & Hérnia; Doenças sexualmente transmissíveis & Raíz, Caule e Folhas & 8.3 \\
\hline Hugenia albyssinica (Bruce) J.F.Gmel. & Dores de estômago; Diarreias; Hérnia & Raíz, Caule e Folhas & 8.3 \\
\hline Pteleopsis myrtifolia (M.A.Lawson) Engl. \& Diels & Dores de estômago & Raíz, Caule e Folhas & 8.3 \\
\hline Zanthoxylum rhetsa (Roxb.) DC & Dores de estômago; Diarreias; DST’s & Raíz, Caule e Folhas & 8.3 \\
\hline Combretum bereroense Schin₹: & Dores de estômago; Malária & Raíz, Caule e Folhas & 8.3 \\
\hline Xylotheca tettensis Gilg, & Dores de estômago; Diarreias & Raíz, Caule e Folhas & 8.3 \\
\hline Adansonia digitata $L$. & Tosse; Dores de estômago & Raíz, Caule, Folhas e Frutos & 4.2 \\
\hline Ricinus communis $L$. & Inflamação; DST's & Raíz e Folhas & 4.2 \\
\hline
\end{tabular}

\section{CONCLUSÕES}

$\mathrm{Na}$ localidade de Nacuale, o uso da medicina tradicional é ainda uma opção viável pela dificuldade de acesso aos serviços de saúde convencional, agravado pelo baixo nível de escolaridade, pobreza e aspectos socioculturais. Neste processo, espécies como a Mangifera indica (Wall), Afizelia quanzensis (Welw), Moringa oleifera (Lam) e Carica papaya (L.) foram citadas como as mais utilizadas para tratamento das doenças mais frequentes na região, como malária, dor de barriga, dor de cabeça e reumatismo.

A maioria dos agregados familiares demostraram ter conhecimento sobre uma considerável diversidade de plantas medicinais actualmente em uso nesta localidade.

\section{REFERÊNCIAS}

AGOSTINHO, A. B. Centro de investigação e de desenvolvimento em etnobotânica: transformando o conhecimento tradicional em científico. Biodiversidade, v. 15, n. 1, p. 67-76, 2016.

AgOSTINHO, A. B.; SILVA, H. L. da. Desafios da medicina tradicional africana no século XXI. In: Congresso Internacional Saber Tropical em Moçambique: História, Memória E Ciência. Anais... Lisboa, 2012. p. 1-9. Disponível em: https://2012congressomz.files.wordpress.com/2013/08 $/ \mathrm{t} 09 \mathrm{c} 01 . \mathrm{pdf}$

BALDIN, N.; MUNHOZ, E. M. B. Snowball (bola de neve): uma técnica metodológica para pesquisa em educação 
ambiental comunitária. In: Congresso Nacional de Educação, X. Anais... Curitiba: PUCPR, 2011. p. 329341. Disponível em: https://educere.bruc.com.br/CD2011/pdf/4398_2342. pdf

BANDEIRA, S.; BOLNICK, D.; BARBOSA, F. Flores nativas do sul de Moçambique. Maputo-Moçambique: Universidade Eduardo Mondlane, 2007. 258p.
BANDEIRA, S.; BARBOSA, F.; BILA, N.; AZEVEDO JR, F.; NACAMO, E.; MANJATE, A. M.; MAFAMBISSA, M.; RAFAEL, J. Terrestrial Vegetation Assessment of the Quirimbas National Park (Final report submitted to the Quirimbas National Park). 2007. 166p. Disponível em: $\quad$ https://www.biofund.org.mz/wpcontent/uploads/2019/01/1548772324-

F0911.Identification $\% 20$ of $\% 20$ all $\% 20$ species $\% 20$.pdf

Tabela 3. Plantas utilizadas pelas populações para tratamento de doenças na localidade de Nacuale no PNQ, Moçambique.

Table 2. Plants used by populations to treat diseases in the locality of Nacuale in PNQ, Mozambique.

\begin{tabular}{|c|c|c|c|}
\hline Família & Nome Científico & Doença / sintoma Curado & Órgão da planta \\
\hline \multirow{4}{*}{ Anacardeaceae } & Anacardium occidentale $L$. & Disenteria & Raiz, Caule e Folhas \\
\hline & Lannea schimperi (Hochst. ex A.Rich.) & Doenças sexualmente transmissíveis (DST’s) & Raiz, Caule e Folhas \\
\hline & Mangifera indica Wall. & Dores de estômago; Malária & Raiz, Caule e Folhas \\
\hline & Sclerocarya birrea Hocbst. & DST's & Raiz, Caule e Folhas \\
\hline Annonaceae & Annona senegalensis Pers. & Dor de Cabeça; Diarreias & Raiz, Caule e Folhas \\
\hline \multirow{2}{*}{ Apocynaceae } & Tabernaemontana elegans Stapf & Tosse; Hérnia & Raiz, Caule e Folhas \\
\hline & Diplorbynchus condylocarpon (Müll.Arg.) Pichon & Dores de estômago & Raiz, Caule e Folhas \\
\hline Bignoniaceae & Kigelia africana (Lam.) Benth. & Desparasitação intestinal & Raiz, Caule e Folhas \\
\hline Bombacaceae & Adansonia digitata $L$. & Tosse; Dores de estomago & Raiz, Caule e Folhas \\
\hline Burseraceae & Commiphora serrata Engl. & Dores de estômago & Raiz, Caule e Folhas \\
\hline Caricaceae & Carica papaya $L$. & Dores de estômago; Diarreias & Raiz, Caule e Folhas \\
\hline \multirow{2}{*}{ Combretaceae } & Pteleopsis myrtifolia (M.A.Lawson) Engl. \& Diels & Dores de estômago & Raiz, Caule e Folhas \\
\hline & Combretum hereroense Schin₹: & Dores de estômago; Malária & Raiz, Caule e Folhas \\
\hline Erythroxylaceae & Erythroxylum emarginatum Thonn. & Disenteria & Raiz, Caule e Folhas \\
\hline Euphorbiaceae & Manibot esculenta Crant: & Dores de estômago; Anemia & Raiz, Caule e Folhas \\
\hline \multirow{19}{*}{ Fabaceae } & Burkea africana Hook. & Diarreias & Raiz, Caule e Folhas \\
\hline & Piliostigma thonningii (Scbumach.) Milne-Redh & Tosse & Raiz, Caule e Folhas \\
\hline & Baubinia thonningii Schumach. \& Thonn & Inflamação; Dores de estômago; Dores de articulações & Raiz, Caule e Folhas \\
\hline & Brachystegia Boehmii Taub. & Desparasitação intestinal & Raiz, Caule e Folhas \\
\hline & Afizelia quanzonsis Welw & Dores de estômago & Raiz, Caule e Folhas \\
\hline & Pisum sativum $L$. & Disenteria; Diarreias & Raiz, Caule e Folhas \\
\hline & Senna Siamea (Lam). & Dores de estômago & Raiz, Caule e Folhas \\
\hline & Acacia Sayal Delile & DST's & Raiz, Caule e Folhas \\
\hline & Milletia stublmanni Taub. & DST's & Raiz, Caule e Folhas \\
\hline & Acássia abreviata Oliver & Diarreias & Raiz, Caule e Folhas \\
\hline & Erythrina abyssinica Lam. ex DC. & Dores de estômago; Dores de dentes & Raiz, Caule e Folhas \\
\hline & Julbernardia globiflora (Benth.) Troupin & Dores de estômago & Raiz, Caule e Folhas \\
\hline & Brachystegia spiciformis (Benth.) & Hérnia & Raiz, Caule e Folhas \\
\hline & Cynomentra carvalhoi Harms. & Hérnia & Raiz, Caule e Folhas \\
\hline & Xeroderris stublmannii Taub. & Dores de estomago; DST's & Raiz, Caule e Folhas \\
\hline & Senna petersiana (Bolle) Lock. & Dores de garganta & Raiz, Caule e Folhas \\
\hline & Acacia polyacantha Willd. & Dores de Cabeça & Raiz, Caule e Folhas \\
\hline & Acácia Senegal (L.). Willd. & Hérnia; DST’s & Raiz, Caule e Folhas \\
\hline & Acacia spp & Tosse & Raiz, Caule e Folhas \\
\hline Kiggelariacea & Xylotheca tettensis Gilg & Dores de estomago; Diarreias & Raiz, Caule e Folhas \\
\hline Linaceae & Hugonia orientalis Engl & Feridas & Raiz, Caule e Folhas \\
\hline Mussadeacea & Musa spp & Dores de estômago; Dores de Cabeça & Raiz, Caule e Folhas \\
\hline \multirow{3}{*}{ Myrtaceae } & Eucalyptus spp & Tosse; Malária & Raiz, Caule e Folhas \\
\hline & Psidium guajava $L$ (GCI) & Dores de estômago; Disenteria; & Raiz, Caule e Folhas \\
\hline & Sysygium cumini (L) Skeels & Malária & Raiz, Caule e Folhas \\
\hline Ricinusdeacea & Ricinus communis $L$. & Inflamação; DST’s & Raiz, Caule e Folhas \\
\hline Moringaceae & Moringa oleifera Lam. & Dores de estomago; Malária; dores de dente & Raiz, Caule e Folhas \\
\hline Phyllanthaceae & Pseudolacbnostylis maprouneifolia Pax. & Epilepsia & Raiz, Caule e Folhas \\
\hline \multirow{2}{*}{ Sterculiaceae } & Sterculia appendiculata K.Schum & Malária & Raiz, Caule e Folhas \\
\hline & Sterculia quimqueloba (Garcke) K. Schum & Bilharziose & Raiz, Caule e Folhas \\
\hline \multirow{2}{*}{ Rubiaceae } & Crossopteryx febrifuga Benth & Dores das articulações; DST’s & Raiz, Caule e Folhas \\
\hline & Xeromphis obovata (Hochst.) Keay & Dores de estômago & Raiz, Caule e Folhas \\
\hline Salicaceae & Flacourtia indica (Burm.f.) Merr & Dores de estômago & Raiz, Caule e Folhas \\
\hline Rutaceae & Zanthoxylum rhetsa (Roxb.) DC & Dores de estômago; Diarreias; DST’s & Raiz, Caule e Folhas \\
\hline Rosaceae & Hagenia albyssinica (Bruce) J.F.Gmel. & Dores de estômago; Diarreias; Hérnia & Raiz, Caule e Folhas \\
\hline
\end{tabular}


BRUMMITT, P. K.; POWELL, C. E. Authors of plant names. A list of authors of scientific names of plants, with recommended standard forms of their names, including abbreviations. Richmond: Royal Botanic Gardens Kew, 1992. 736p.

CASAGRANDE, A. Plantas medicinais e ritualísticas utilizadas pela comunidade do Morro da Cruz, Porto Alegre-RS. 91p. [Trabalho de Conclusão de Curso Ciências Biológicas] - Universidade Federal do Rio Grande do Sul, Porto Alegre, 2009. Disponível em: https://lume.ufrgs.br/handle/10183/18661

CASTELLUCCI, S.; LIMA, M. I.; NORDI, N.; MARQUES, J. G. W. Plantas medicinais relatadas pela comunidade residente na estação ecológica de Jataí, município de Luís António/SP: uma abordagem Etnobotânica. Revista Brasileira de Plantas Medicinais, v. 3, n. 1, p. 51-60, 2000.

CASTRO, H. G.; FERREIRA, F. A. A dialéctica do conhecimento no uso das plantas medicinais. Revista Brasileira de Plantas Medicinais, v. 3, n. 2, p. 19-21, 2001.

CLIFF, J.; SACARLAL, J.; AUGUSTO, O.; NÓVOA, A.; DGEDGE, M.; MACHATINE, G.; COSSA, H. Estudo das principais causas de morte registadas, nas cidades de Maputo, Beira, Chimoio e Nampula, em 2001 (Relatório Final). Ministério da Saúde Faculdade de Medicina-UEM, Centro de Investigação em Saúde da Manhiça, 2003. 44p.

CONDE, P.; FIGUEIRA, R.; SARAIVA, S.; CATARINO, L.; ROMEIRAS, M.; DUARTE, M. C. A Missão Botânica de Moçambique (1942-1948): contribuições para o conhecimento da flora medicinal de Moçambique. História, Ciências, Saúde-Manguinhos, v. 21, n. 2, p. 539-585, 2014

CUNNINGHAM, A. B. African medicinal plants: setting priorities at the interface between conservation and primary healthcare. Paris-UNESCO: People and Plants Working Paper, n. 1, 53p. Disponível em: https:// citeseerx.ist.psu.edu/viewdoc/download?doi=1 0.1.1.294.2667\&rep $=$ rep1\&type $=$ pdf

DA SILVA, G.; GOMES, E. T.; SILVA, O. Estudos fotoquímicos e de actividade biológica de espécies moçambicanas do género Maytenus. In: Workshop Plantas Medicinais e Praticas Fitoterapêuticas nos Trópicos. Anais... Lisboa: Instituto de Investigação Científica Tropical, 2009. 6p.

DE MELLO AMOROZO, M. C. Uso e diversidade de plantas medicinais em Santo António do Leverger, MT, Brasil. Acta Botanica Brasilica, v. 16, n. 2, p. 189-203, 2002.

DHARANI, N.; YENESEW, A. Medicinal plants of east Africa - an illustrated guide. Nairobi: Drongo Editing \& Pub., 2010. 272p.

DHARANI, N. Field guide to common Medicinal plants of east Africa. Trees e shrubs of east Africa. Southern Africa: Struik Nature, 2002. 384p.

ERNESTO, M. M. Composição Química de extractos da Momordica balsamina cultivada em Moçambique. 95f. Dissertation [Doctoral] - Universidade de Aveiro, 2006. Disponível

em https://ria.ua.pt/bitstream/10773/4863/1/2007001156 .pdf
FONSECA-KRUEL, V. S. D.; PEIXOTO, A. L. Etnobotânica na reserva extractivista marinha de Arraial do Cabo, RJ, Brasil. Acta Botânica Brasilica, v. 18, p. 177-190, 2004. DOI: https://doi.org/10.1590/S010233062004000100015

GIRALDI, M.; HANAZAKI, N. Uso e conhecimento tradicional de plantas medicinais no Sertão do Ribeirão, Florianópolis, SC, Brasil. Acta Botanica Brasilica, v. 24, p. 395-406, 2010.

HOSTETTMANN, K.; MARTSON, A.; NDJOKO, K.; WOLFENDER, J.-L. The potential of African plants as a source of drugs. Current Organic Chemistry, v. 4, n. 10, p. 973-1010, 2000. DOI: 10.2174/1385272003375923

INE_Instituto Nacional de Estatística. Estatística do distrito de Ancuabe. 2013. Disponível em: http://www.ine.gov.mz/estatisticas/estatisticasterritorias-distritais/cabodelgado/2012/marco/estatisticas-do-distrito-deancuabe.pdf/view

MAZIVE, E.; NHAPURE, G.; DUCE, P. Mortalidade em Moçambique inquérito nacional sobre causas de mortalidade, 2007/8. Maputo, Mozambique: Instituto Nacional de Estatística. 2009. 100p. Disponível em: http:/ /www.ine.gov.mz/operacoes-

estatisticas/inqueritos/inquerito-sobre-causas-demortalidade

MAE_Ministério Da Administração Estatal. Perfil do distrito de Ancuabe, Província de Cabo Delgado. 2014. 61p. Disponível

http://www.ine.gov.mz/documentos/outrosdocumentos/perfil-do-distrito-de-ancuabe-cabodelgado.pdf/view

MONTEIRO, J. Amostragem probabilística e não probabilística: técnicas e aplicações na determinação de amostras. Jerónimo Monteiro: UFES/CCA, 2012. 28p.

NANVONAMUQUITXO, S. J. A.; MACUEIA, F. B. E. D.; CARAVELA, M. I. Estrutura e diversidade de uma floresta de Miombo em Taratibu, Norte de Moçambique. Nativa, v. 7, n. 6, p. 778-783, 2019. DOI: http://dx.doi.org/10.31413/nativa.v7i6.7198.

OLSON, D. M.; DINERSTEIN, E.; WIKRAMANAYAKE, E. D.; BURGESS, N. D.; POWELL, G. V.; UNDERWOOD, E. C.; LOUCKS, C. J. Terrestrial Ecoregions of the World: A New Map of Life on Earth A new global map of terrestrial ecoregions provides an innovative tool for conserving biodiversity. Bio Science, v. 51, n. 11, p. 933-938, 2001.

PINTÃO, A. M.; SILVA, I. F. D. A verdade sobre o açafrão. In: Workshop Plantas Medicinais e Fitoterapêuticas nos Trópicos. Anais... Portugal: IICT/CCCM, v. 29, n. 30, p. 1-19, 2008.

PINTO, E. D. P. P.; AMOROZO, M. C. D. M.; FURLAN, A. Conhecimento popular sobre plantas medicinais em comunidades rurais de mata atlântica-Itacaré, BA, Brasil. Acta Botânica Brasilica, v. 20, p. 751-762, 2006.

PNQ. (Ed.). Caracterização dos distritos abrangidos pelo parque nacional das Quirimbas. Maputo: relatório do estudo sócio-económico, 2016. 99p.

RICHARDSON, R. J.; PERES, J. A. S.; WANDERLEY, J. C. V.; CORREIA, L. M.; PERES, M. H. M. Pesquisa 
social: Métodos e técnicas. 3 ed. Editora Atlas S.A. São Paulo. 1999. 54p.

SANTOS, J. J. F.; COELHO-FERREIRA, M.; LIMA, P. G. C. Etnobotânica de plantas medicinais em mercados públicos da Região Metropolitana de Belém do Pará, Brasil. Biota Amazônia, v. 8, n. 1, p. 1-9, 2018.

SERRANO, R.; GOMES, E. T.; SILVA, O. Identificação botânica de Maytenus senegalensis folha como fármaco vegetal. In: Workshop Plantas Medicinais e Fitoterapêuticas nos Trópicos. Proceedings... Portugal: IICT/CCCM, 2008. 7p.

SILVA, J. R. D. A.; RAMOS, A. D. S.; MACHADO, M.; DE MOURA, D. F.; NETO, Z.; CANTO-CAVALHEIRO, M. M.; LOPES, D. A review of antimalarial plants used in traditional medicine in communities in Portuguesespeaking countries: Brazil, Mozambique, Cape Verde, Guinea-Bissau, São Tomé and Príncipe and Angola. Memorias do Instituto Oswaldo Cruz, v. 106, p. 142 158, 2011.

SIMBINE, M. D. G. Z. Factores antrópicos e conservação da Floresta Sagrada de Chirindzene, Gaza-Moçambique. 70f. Dissertação [Mestrado Ecologia, Ambiente e Território] - Universidade do Porto, 2013. Disponível em: https://repositorioaberto.up.pt/bitstream/10216/67495/2/24443.pdf

THE ANGIOSPERM PHYLOGENY GROUP; CHASE, M. W.; CHRISTENHUSZ, M. J. M.; FAY, M. F.; BYNG, J. W.; JUDD, W. S.; SOLTIS, D. E.; MABBERLEY, D. J.; SENNIKOV, A. N.; SOLTIS, P. S.; STEVENS, P. F. An update of the Angiosperm Phylogeny Group classification for the orders and families of flowering plants: APG IV. Botanical Journal of the Linnean Society, v. 181, n. 1, p. 1-20, 2016. DOI: https://doi.org/10.1111/boj.12385.

VAN WYK, B.; VAN WYK, P. Field Guide to Trees of Southern Africa. South Africa: Penguin Random House South Africa, 2013. 720p.

VENDRUSCOLO, G. S.; MENTZ, L. A. Estudo da concordância das citações de uso e importância das espécies e famílias utilizadas como medicinais pela comunidade do bairro Ponta Grossa, Porto Alegre, RS, Brasil. Acta Botânica Brasilica, v. 20, p. 367-382, 2006. DOI: $\quad$ https://doi.org/10.1590/S010233062006000200012 .

VINUTO, J. A amostragem em bola de neve na pesquisa qualitativa: um debate em aberto. Temáticas, v. 22, n. 44, p. 203-220, 2014. DOI: https://doi.org/10.20396/tematicas.v22i44.10977.

WHO_World Health Organization. Traditional medicine strategy, 2002-2005. Genebra. 2002. Disponível em: http://apps.who.int/medicinedocs/en/d/Js4930s/. Acessado em 8 mar. 2018. 